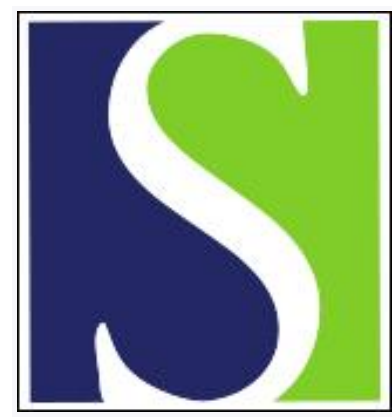

Scand J Work Environ Health 2012;38(5):436-446

https://doi.org/10.5271/sjweh.3285

Published online: 27 Feb 2012, Issue date: 01 Sep 2012

Risk factors for incidence of rotator cuff syndrome in a large working population

by Bodin J, Ha C, Petit Le Manac'h A, Sérazin C, Descatha A, Leclerc A, Goldberg M, Roquelaure Y

Affiliation: Laboratoire d'ergonomie et d'épidémiologie en santé au travail, Faculté de Médecine, Rue Haute de Reculée, 49045 ANGERS Cedex 01, France. julie.bodin@univ-angers.fr

Refers to the following texts of the Journal: 2011;37(6):502-511 2010;36(3):189-201 2009;35(2):113-126 2006;32(4):294-299 2001;27 suppl 1:1-102 2006;32(2):99-108

The following articles refer to this text: $2013 ; 39(6): 568-577$; 2020;46(6):618-629; 2022;48(6):490-497; 2023;49(1):53-63

Key terms: incidence; MSD; musculoskeletal disease; occupational factor; personal risk factor; RCS; risk factor; rotator cuff syndrome; shoulder; tendinitis; work; work; work; working population

This article in PubMed: www.ncbi.nlm.nih.gov/pubmed/22367342 


\title{
Risk factors for incidence of rotator cuff syndrome in a large working population
}

\author{
by Julie Bodin, MsC, ${ }^{2}$ Catherine Ha, MD, PhD, ${ }^{2}$ Audrey Petit Le Manac'h, MD, ${ }^{1}$ Céline Sérazin, MsC, ${ }^{1}$ Alexis \\ Descatha, MD, PhD, ${ }^{3}$ Annette Leclerc, PhD, ${ }^{3}$ Marcel Goldberg, MD, PhD, ${ }^{3}$ Yves Roquelaure, MD, PhD ${ }^{1}$
}

\begin{abstract}
Bodin J, Ha C, Petit Le Manac'h A, Sérazin C, Descatha A, Leclerc A, Goldberg M, Roquelaure Y. Risk factors for incidence of rotator cuff syndrome in a large working population. Scand J Work Environ Health. 2012;38(5):436446. doi:10.5271/sjweh.3285
\end{abstract}

Objectives The aim of this study was to assess the effects of personal and work-related factors on the incidence of rotator cuff syndrome (RCS) in a large working population.

Methods A total of 3710 French workers were included in a cross-sectional study in 2002-2005. All completed a self-administered questionnaire about personal factors and work exposure. Using a standardized physical examination, occupational physicians established a diagnosis of RCS. Between 2007-2010, 1611 workers were re-examined. Associations between RCS and risk factors at baseline were analyzed by logistic regression.

Results A total of 839 men and 617 women without RCS at baseline were eligible for analysis. RCS was diagnosed in 51 men $(6.1 \%)$ and 45 women $(7.3 \%)$. The risk of RCS increased with age for both genders [odds ratio (OR) $4.7(95 \%$ confidence interval $[95 \% \mathrm{CI}] 2.2-10.0)$ for men aged $45-49$ years and $5.4(95 \% \mathrm{CI} 2.3-13.2)$ for women aged 50-59 years; reference $<40$ years]. For men, the work-related risk factors were repeated posture with the arms above the shoulder level combined with high perceived physical exertion [OR $3.3(95 \%$ CI 1.3-8.4)] and low coworker support [OR $2.0(95 \% \mathrm{CI} 1.1-3.9)]$. For women, working with colleagues in temporary employment [OR $2.2\left(95 \%\right.$ CI 1.2-4.2)] and repeated arm abduction $\left(60-90^{\circ}\right)$ [OR $2.6(95 \%$ CI 1.4-5.0)] were associated with RCS.

Conclusions Age was the strongest predictor for incident cases of RCS, and arm abduction was the major workrelated risk factor for both genders. Lack of social support was a predictor for RCS among men.

Key terms musculoskeletal disease; MSD; occupational factor; personal risk factor; RCS; shoulder; tendinitis; work.

Rotator cuff syndrome (RCS) is a common upperextremity musculoskeletal disorder (MSD) in the working population and causes long periods of absence from work (1-7). The physiopathology of RCS involves degenerative changes in the rotator cuff tendons, compression of the tendons between the humeral head and the coracoacromial arch, and ischemia due to impingement or increased intramuscular pressure (8).

Several studies have shown that the prevalence of RCS increased with age (9-11) and some suggested a link between RCS and both individual factors, such as diabetes mellitus or abdominal obesity $(9,10,12$, $13)$, and work-related factors $(1-4,10,11,14-18)$. A recent review of the literature reported that RCS was associated with frequent handling of loads or with high force, highly repetitive work, and work above shoulder level, whereas the association with hand-arm vibration remained unclear (19). High job demand was associated with RCS, but the link between RCS and psychosocial factors differed according to the studies $(2,3,5,10,19)$. However, most studies were based on cross-sectional design, and there is a need for prospective studies to confirm these associations.

Using the data of the surveillance program for upper extremity MSD implemented by the French Institute for Public Health Surveillance in the Pays de la Loire region, we have previously studied the personal and work-related risk factors associated with prevalent cases

1 LUNAM, Université d'Angers, LEEST, Angers, France.

2 Département santé travail, Institut de veille sanitaire (DST-InVS), Saint-Maurice, France.

3 INSERM, U1018, Villejuif, France.

Correspondence to: Julie Bodin, Laboratoire d'ergonomie et d'épidémiologie en santé au travail, Faculté de Médecine, Rue Haute de Reculée, 49045 ANGERS Cedex 01, France. [E-mail: julie.bodin@univ-angers.fr] 
of RCS occurring between 2002-2005 (7). The aim of this prospective study was to assess the effects of personal and work-related factors on the incidence of RCS among a large working population.

\section{Methods}

\section{Study population}

This prospective study was based on two successive surveys of a large sample of workers in the French Pays de la Loire region. The region contains $5.6 \%$ of the French working population, and its socioeconomic structure is close to that of France as a whole (20).

At the time of the first survey, all French salaried workers, including temporary and part-time workers, underwent a mandatory annual health examination by an occupational physician (OP) in charge of the medical surveillance of a group of companies. Between $2002-2005,83$ OP (18\% of OP of the region) volunteered to take part in the study. They were representative of the region's OP in terms of working time and geographic and economic sectors covered (21). Subjects were selected at random, following a two-stage sampling procedure: first, the research team chose 15-45 halfdays of scheduled examinations for each OP. Next, using random sampling tables, each OP selected 1 out of 10 workers from the schedule on the half-days of worker examinations considered. A total of 3710 workers [2161 men (58.2\%), mean age 38.5 (standard deviation [SD] 10.4) years and 1549 women (42.8\%), mean age 38.9 (SD 10.3) years] were included. The distribution of occupations in the study sample was close to that of the regional workforce (20-22).

Medical follow-up of the workers initially included was undertaken between 2007-2010. Retired people, people on parental or long-term sick leave, and unemployed people were excluded. Several reminders were sent out to all occupational medicine services, and then to each OP now responsible for the medical surveillance of at least one worker of the cohort. For workers who had changed $\mathrm{OP}$, the research team systematically contacted the last OP responsible for their medical surveillance. After approval, each OP received guidelines describing the standardized clinical procedure that strictly applied the methodology and clinical tests of the European consensus criteria document for RCS and the five other upper extremity MSD surveyed (lateral epicondylitis, ulnar tunnel syndrome, carpal tunnel syndrome, De Quervain's disease and flexorextensor peritendinitis, or tenosynovitis of the forearmwrist region) (23). The research team conducted a training program for the OP to standardize physical examinations at baseline and follow-up.

\section{Outcome}

"Incident cases" of RCS were defined as workers free of RCS at baseline with diagnosed RCS at follow-up. Thus cases occurring between the two data collection periods that were negative at follow-up were not considered as incident cases.

In cases of upper-limb musculoskeletal symptoms occurring during the preceding 12 months, the OP conducted a physical examination to diagnose RCS and the five other upper extremity MSD surveyed. RCS was diagnosed if (i) there was at least intermittent pain in the shoulder region (without paresthesia), worsened by active elevation movement of the upper arm, as in scratching the upper back, currently or for $\geq 4$ days during the preceding 7 days; and (ii) if $\geq 1$ of the following shoulder tests was positive: resisted shoulder abduction, external or internal rotation; resisted elbow flexion (palm-up test); and painful arc on active upper arm test (abduction-elevation).

\section{Potential risk factors}

At baseline, workers completed a self-administered questionnaire about their musculoskeletal symptoms and their working conditions during a typical working day during the 12 preceding months and then underwent a physical examination. Potential risk factors were divided into four groups. The personal factors studied were age, body mass index (BMI) and upper-extremity MSD at baseline (defined as the diagnosis of $\geq 1$ of the five other upper-extremity MSD). Work-related factors were divided into three groups.

Work organization. The factors related to the work organization were: time constraints (paced work, work pace dependent on automatic rate, colleagues' work, quantified targets, customers' demand, permanent controls, or surveillance), job/task rotation, overtime hours, work with temporary workers, high visual demand, lack of prior information regarding the amount of work to be done each day, variable weekly working time, working with temporary workers and temporary employment.

Biomechanics. The biomechanical factors were assessed using the European consensus criteria document for evaluation of the work-relatedness of upper-extremity MSD (23): high repetitiveness of tasks ( $\geq 4$ hours/day), repeated and sustained posture with the arms above shoulder level ( $\geq 2$ hours/day) and holding the hand behind the trunk ( $\geq 2$ hours/day). Workers were defined as being at risk of working with repeated and sustained arm abduction $\left(60-90^{\circ}\right)$ if they responded "rarely $(<2$ hours/day)", "often (2-4 hours/day)" or "always ( $\geq 4$ hours/day)". Additional variables, known or suspected to be risk factors, were considered: use of hand tools 
( $\geq 2$ hours/day), use of vibrating hand tools ( $\geq 2$ hours/ day) and exposure to cold temperature ( $\geq 4$ hours/day). Perceived physical exertion was assessed using the Borg rating of perceived exertion (RPE) scale graduated from 6 ("very, very light") to 20 ("maximum exertion"). High perceived physical exertion was dichotomized using the third quartile ( $\geq 15$ for men and $\geq 14$ for women) $(24,25)$.

Psychosocial work factors. The psychosocial work factors were assessed using the validated French version of Karasek's Job Content Questionnaire. Scores were dichotomized using the median scores of the national French SUMER (medical surveillance of occupational risk exposures) study (26).

\section{Statistical analysis}

Analyses were performed separately for men and women to take into account gender differences in exposure to work constraints (27).

Associations between RCS and the risk factors were studied by Chi-squared or Fisher's exact tests. Risk factors with a P-value $<0.20$ were introduced in a multivariable model by groups of variables. Manual backward multivariate logistic regression models were then applied for each group in order to avoid colinearity between exposure variables in the final model (age was forced into the models). The remaining factors $(\mathrm{P}<0.10)$ were entered into a final global multivariate logistic regression model and manual backward selection retained only significant variables with a P-value of 0.05. In manual backward multivariate logistic regression, if there was a change in the beta coefficients of $\geq 15 \%$ when a variable was deleted, this variable was considered as a confounder and was forced into the model. The Hosmer and Lemeshow test was used to determine goodness-of-fit of the logistic model.

Finally, the whole modeling process was performed again by excluding workers with RCS, workers with shoulder pain for $>1$ month at baseline, and workers with missing values.

All analyses were performed with the statistical software package SAS (version 9.2, SAS Institute Inc, Cary, NC, USA). Each subject provided informed written consent to participate in this study at baseline and the study received approval from France's National Committee for Data Protection (Commission Nationale de l'Informatique et des Libertés), first in 2001 and again in 2006.

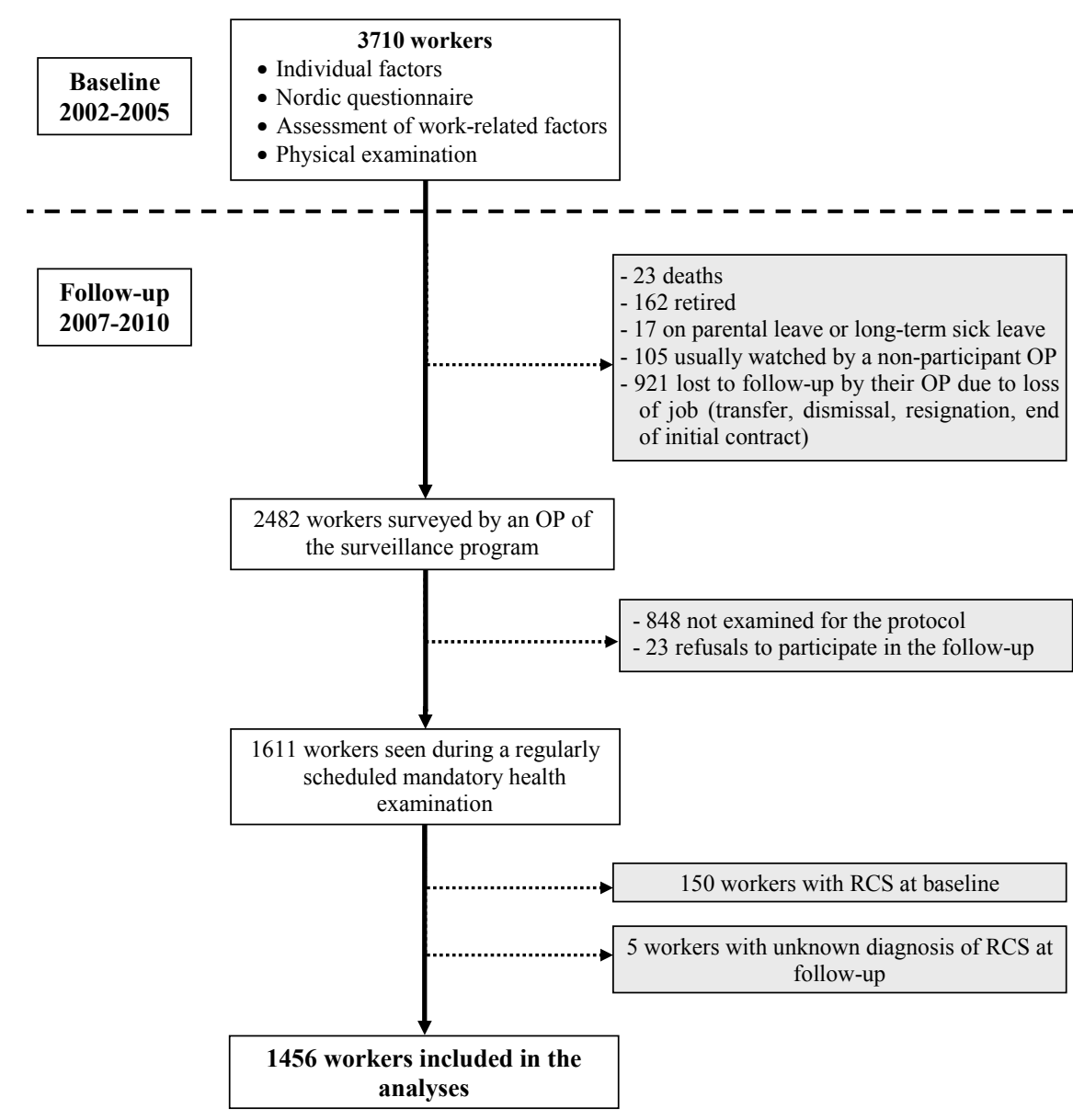

Figure 1. Study population flowchart. [RCS=rotator cuff syndrome] 
Table 1. Comparison of baseline characteristics of workers with follow-up and workers without follow-up, according to gender.

\begin{tabular}{|c|c|c|c|c|c|c|c|c|c|c|}
\hline & \multicolumn{5}{|c|}{ Men $(\mathrm{N}=2161)$} & \multicolumn{5}{|c|}{ Women $(\mathrm{N}=1549)$} \\
\hline & \multicolumn{2}{|c|}{$\begin{array}{l}\text { With follow-up } \\
(\mathrm{N}=921)\end{array}$} & \multicolumn{2}{|c|}{$\begin{array}{l}\text { Without follow-up } \\
\qquad(\mathrm{N}=1240)\end{array}$} & \multirow[b]{2}{*}{ P-value } & \multicolumn{2}{|c|}{$\begin{array}{l}\text { With follow-up } \\
(\mathrm{N}=690)\end{array}$} & \multicolumn{2}{|c|}{$\begin{array}{l}\text { Without follow-up } \\
\qquad(N=859)\end{array}$} & \multirow[b]{2}{*}{ P-value } \\
\hline & $\mathrm{N}$ & $\%$ & $\mathrm{~N}$ & $\%$ & & $\mathrm{~N}$ & $\%$ & $\mathrm{~N}$ & $\%$ & \\
\hline \multicolumn{11}{|l|}{ Personal factors and medical history } \\
\hline Age & & & & & $<0.001$ & & & & & $<0.001$ \\
\hline$<40$ & 473 & 51.4 & 669 & 54.0 & & 316 & 45.8 & 466 & 54.3 & \\
\hline $40-44$ & 180 & 19.5 & 137 & 11.1 & & 132 & 19.1 & 112 & 13.0 & \\
\hline $45-49$ & 161 & 17.5 & 142 & 11.5 & & 140 & 20.3 & 91 & 10.6 & \\
\hline$\geq 50$ & 107 & 11.6 & 291 & 23.5 & & 102 & 14.8 & 190 & 22.1 & \\
\hline Overweight or obesity ( $\geq 30$ kg/m²) & 394 & 43.4 & 536 & 43.6 & 0.919 & 185 & 27.2 & 263 & 31.2 & 0.093 \\
\hline Upper-extremity musculoskeletal disorders a & 62 & 6.7 & 69 & 5.6 & 0.261 & 55 & 8.0 & 71 & 8.3 & 0.833 \\
\hline $\begin{array}{l}\text { Shoulder pain lasting } \geq 1 \text { day during the } \\
\text { preceding } 12 \text { months }\end{array}$ & 294 & 31.9 & 440 & 35.5 & 0.084 & 262 & 38.0 & 340 & 39.6 & $0.458^{b}$ \\
\hline $\begin{array}{l}\text { Shoulder pain lasting }>1 \text { month during the } \\
\text { preceding } 12 \text { months }\end{array}$ & 73 & 8.0 & 124 & 10.1 & 0.093 & 86 & 12.8 & 116 & 13.7 & 0.581 \\
\hline $\begin{array}{l}\text { Shoulder pain lasting } \geq 1 \text { day during the } \\
\text { preceding } 7 \text { days }\end{array}$ & 152 & 16.5 & 202 & 16.3 & 0.905 & 147 & 21.4 & 183 & 21.4 & 0.995 \\
\hline \multicolumn{11}{|l|}{ Work history } \\
\hline Number of years in the initial job & & & & & $<0.001$ & & & & & $<0.001$ \\
\hline$<1$ & 73 & 8.0 & 197 & 16.1 & & 57 & 8.3 & 128 & 15.1 & \\
\hline $1-2$ & 133 & 14.6 & 201 & 16.4 & & 102 & 14.9 & 155 & 18.3 & \\
\hline $3-10$ & 333 & 36.5 & 392 & 32.0 & & 240 & 35.0 & 273 & 32.2 & \\
\hline$>10$ & 373 & 40.9 & 436 & 35.6 & & 287 & 41.8 & 293 & 34.5 & \\
\hline Occupational category & & & & & 0.854 & & & & & 0.135 \\
\hline Manager, professional, technician & 332 & 36.1 & 431 & 34.8 & & 166 & 24.1 & 204 & 23.8 & \\
\hline Low-grade white collar & 81 & 8.8 & 106 & 8.6 & & 374 & 54.3 & 425 & 49.6 & \\
\hline Skilled blue collar & 353 & 38.4 & 479 & 38.7 & & 43 & 6.2 & 68 & 7.9 & \\
\hline Unskilled blue collar & 154 & 16.7 & 223 & 18.0 & & 106 & 15.4 & 160 & 18.7 & \\
\hline Economic sector & & & & & $<0.001$ & & & & & 0.008 \\
\hline Agriculture & 8 & 0.9 & 23 & 1.9 & & 3 & 0.4 & 22 & 2.6 & \\
\hline Industries & 425 & 46.3 & 434 & 35.1 & & 178 & 25.9 & 223 & 26.0 & \\
\hline Construction & 71 & 7.7 & 118 & 9.5 & & 9 & 1.3 & 16 & 1.9 & \\
\hline Trade and services & 415 & 45.2 & 662 & 53.5 & & 498 & 72.4 & 596 & 69.5 & \\
\hline Temporary employment & 53 & 5.8 & 183 & 14.8 & $<0.001$ & 40 & 5.8 & 150 & 17.6 & $<0.001$ \\
\hline \multicolumn{11}{|l|}{ Factors related to work organization } \\
\hline Paced work & 101 & 11.2 & 134 & 11.1 & 0.944 & 48 & 7.2 & 100 & 12.1 & 0.002 \\
\hline Work pace dependent on automatic rate & 113 & 12.5 & 145 & 12.0 & 0.737 & 47 & 7.1 & 95 & 11.4 & 0.004 \\
\hline Work pace dependent on colleagues' work & 284 & 31.6 & 414 & 34.5 & 0.175 & 159 & 23.9 & 252 & 30.4 & 0.006 \\
\hline Work with temporary workers & 265 & 28.8 & 374 & 30.2 & 0.504 & 183 & 26.6 & 284 & 33.3 & 0.004 \\
\hline \multicolumn{11}{|c|}{ Working postures and biomechanical constraints } \\
\hline High perceived physical exertion ${ }^{c}$ & 192 & 21.0 & 311 & 25.2 & 0.024 & 146 & 21.2 & 203 & 23.9 & 0.215 \\
\hline $\begin{array}{l}\text { Repeated and sustained posture with the arms } \\
\text { above the shoulder ( } \geq 2 \mathrm{~h} / \text { day) }\end{array}$ & 113 & 12.3 & 193 & 15.7 & 0.027 & 77 & 11.2 & 104 & 12.1 & 0.588 \\
\hline $\begin{array}{l}\text { Repeated and sustained arm abduction } \\
\left(60-90^{\circ}\right)\end{array}$ & 332 & 36.1 & 506 & 41.1 & 0.019 & 193 & 28.1 & 225 & 26.3 & 0.419 \\
\hline Holding hand behind the trunk ( $\geq 2 \mathrm{~h} /$ day) & 37 & 4.0 & 73 & 5.9 & 0.047 & 38 & 5.5 & 39 & 4.6 & 0.386 \\
\hline \multicolumn{11}{|l|}{ Psychosocial factors at work } \\
\hline Low coworker support & 162 & 17.7 & 244 & 20.1 & 0.168 & 118 & 17.5 & 184 & 22.0 & 0.033 \\
\hline
\end{tabular}

\section{Results}

\section{Participation in follow-up}

Of the 83 OP who participated at baseline, 60 surveyed at least one of the 3710 workers between 2007-2010.
Of the OP who did not participate in the baseline study, 94 became responsible for the medical surveillance of at $\geq 1$ worker and 85 agreed to participate. A total of 1228 workers was excluded (death, retirement, parental leave, long-term sick leave, unemployment, etc). Of the 2482 remaining workers, 23 refused to participate 
Table 2. Baseline characteristics of workers.

\begin{tabular}{|c|c|c|c|c|c|}
\hline \multirow[t]{2}{*}{ Baseline characteristics } & \multicolumn{2}{|c|}{$\begin{array}{c}\text { Men } \\
(\mathrm{N}=839)\end{array}$} & \multicolumn{2}{|c|}{$\begin{array}{l}\text { Women } \\
(\mathrm{N}=617)\end{array}$} & \multirow[t]{2}{*}{ P-value a } \\
\hline & $\mathrm{N}$ & $\%$ & $\mathrm{~N}$ & $\%$ & \\
\hline Age & & & & & 0.158 \\
\hline$<40$ & 449 & 53.5 & 299 & 48.5 & \\
\hline $40-44$ & 164 & 19.6 & 120 & 19.5 & \\
\hline $45-49$ & 137 & 16.3 & 116 & 18.8 & \\
\hline$\geq 50$ & 89 & 10.6 & 82 & 13.3 & \\
\hline $\begin{array}{l}\text { Shoulder pain lasting } \geq 1 \text { day } \\
\text { during the preceding } 12 \text { months }\end{array}$ & 217 & 25.9 & 192 & 31.2 & 0.026 \\
\hline $\begin{array}{l}\text { Shoulder pain lasting }>1 \text { month } \\
\text { during the preceding } 12 \text { months }\end{array}$ & 38 & 4.6 & 52 & 8.6 & 0.002 \\
\hline $\begin{array}{l}\text { Shoulder pain lasting } \geq 1 \text { day } \\
\text { during the preceding } 7 \text { days }\end{array}$ & 89 & 10.6 & 83 & 13.5 & 0.096 \\
\hline $\begin{array}{l}\text { Intensity of shoulder pain } \\
\text { during the preceding } 7 \text { days } \\
\text { (scale } 0-10)\end{array}$ & & & & & 0.221 \\
\hline $0-1$ & 14 & 16.1 & 12 & 15.0 & \\
\hline $2-4$ & 47 & 54.0 & 34 & 42.5 & \\
\hline $5-10$ & 26 & 29.9 & 34 & 42.5 & \\
\hline Occupational class & & & & & $<0.001$ \\
\hline $\begin{array}{l}\text { Manager, professional, } \\
\text { technician }\end{array}$ & 306 & 36.5 & 153 & 24.8 & \\
\hline Low-grade white collar & 76 & 9.1 & 341 & 55.4 & \\
\hline Skilled blue collar & 320 & 38.2 & 34 & 5.5 & \\
\hline Unskilled blue collar & 136 & 16.2 & 88 & 14.3 & \\
\hline Economic sector & & & & & $<0.001^{b}$ \\
\hline Agriculture & 8 & 1.0 & 3 & 0.5 & \\
\hline Industry & 389 & 46.5 & 148 & 24.0 & \\
\hline Construction & 65 & 7.8 & 8 & 1.3 & \\
\hline Trade and services & 375 & 44.8 & 457 & 74.2 & \\
\hline
\end{tabular}

a Comparison of baseline characteristics between men and women, $\chi^{2}$ test.

${ }^{\mathrm{b}}$ Fisher exact test.

and 848 workers did not undergo the second physical examination because they had no mandatory examination scheduled between the moment the OP learned that he/she was responsible for a worker and the end of the follow-up period. Finally, 1611 workers $(64.9 \%)$ were re-examined (figure 1).

The follow-up rate did not differ with gender $(42.6 \%$ for men versus $44.5 \%$ for women) or baseline occupational category.

A comparison between the group of workers with a follow-up and the group of workers lost to follow-up is presented in table 1 . There was a significant difference in age between the workers who were followed up and those lost to follow-up: workers aged $<40$ years and those aged $>50$ years were more frequent in the group of workers lost to follow-up. Moreover, workers with length of service of $<2$ years and temporary workers were more frequent among the workers lost to follow-up. For men, exposure to high perceived physical exertion, arms above shoulder level, arm abduction $\left(60-90^{\circ}\right)$ and holding the hand behind the trunk differed between the two groups, such exposure being more frequent for workers lost to
Table 3. Incidence of rotator cuff syndrome (RCS) according to personal and work-related factors. [MSD=musculoskeletal disorder]

\begin{tabular}{|c|c|c|c|}
\hline \multicolumn{3}{|c|}{ Men $(\mathrm{N}=839)$} & Women $(\mathrm{N}=617)$ \\
\hline $\mathrm{N}$ & $\begin{array}{l}\text { Incidence } \\
(\%)\end{array}$ & $\begin{array}{c}\mathrm{P}- \\
\text { value }\end{array}$ & $\begin{array}{c}\text { N Incidence } \\
(\%) \quad \text { value }^{a}\end{array}$ \\
\hline
\end{tabular}

Personal factors and medical history

Age (years)

$<40$

$40-44$

$45-49$

$\geq 50$

Body mass index

Underweight or

normal $\left(<25 \mathrm{~kg} / \mathrm{m}^{2}\right)$

Overweight (25-30

$\left.\mathrm{kg} / \mathrm{m}^{2}\right)$

Obesity $\left(\geq 30 \mathrm{~kg} / \mathrm{m}^{2}\right)$

Upper-extremity

MSD ${ }^{c}$

No

Yes

(\%) value ${ }^{a}$

Factors related to work

organization

Paced work

No

Yes

Work pace dependent

on automatic rate

No

Yes

Work pace dependent on other technical

organization

No

Yes

Work pace dependent

on colleagues' work

No

Yes

Work pace dependent

on quantified targets

No

Yes

Work pace dependent

on demand of

customers

No

Yes

$449 \quad 31$

$164 \quad 6.7$

$<0.001$

$137 \quad 12.4$

$89 \quad 10.1$

$475 \quad 4.8$

295

8.8

$60 \quad 1.7$

1.7

$<0.001$

299

3.7

0.003

$797 \quad 5.8$

$42 \quad 11.9$

$\begin{array}{ll}120 & 8.3\end{array}$

$\begin{array}{ll}116 & 10.3\end{array}$

$82 \quad 14.6$

0.027

$451 \quad 6.0$

0.081

$110 \quad 11.8$

$49 \quad 10.2$

$0.171^{\mathrm{b}}$

0.179

$\begin{array}{ll}580 & 6.9\end{array}$

$37 \quad 13.5$

0.454

$734 \quad 5.9$

$89 \quad 7.9$

0.454

$555 \quad 7.0$

$38 \quad 7.9$
$0.974 \quad 0.0$

$0.745^{b}$

(19)

$725 \quad 6.2$

$98 \quad 6.1$

$\begin{array}{ll}559 & 6.8\end{array}$

$36 \quad 13.9$

$0.608^{b}$

$596 \quad 6.5$

$226 \quad 4.9$

$\begin{array}{rrr}527 & 7.4 \\ & 65 & 4.6 \\ 0.674 & & \end{array}$

0.449

$570 \quad 6.3$

$452 \quad 6.6$

$252 \quad 5.6$

$141 \quad 8.5$

0.400

0.244

$394 \quad 5.3$

381

6.3

$431 \quad 6.7$

0.104

Work pace dependent

on permanent controls

or surveillance

No

Yes

456

$372 \quad 7.5$

308

8.1

$297 \quad 5.7$

Job/task rotation ( $\geq 1$

job rotation per week)

No

Yes

Overtime hours

No

Yes

607

6.3

463

6.1

$216 \quad 5.6$

$130 \quad 11.5$

0.181

0.661

$491 \quad 7.1$

$313 \quad 4.8$

$\begin{array}{ll}381 & 6.8\end{array}$

$205 \quad 7.8$

0.944

0.097

Work with temporary

$285 \quad 6.0$

$542 \quad 6.1$

workers

No
Yes
High visual demand
No

No

241

5.7

$331 \quad 5.7$ 
Table 3. Continued

\begin{tabular}{|c|c|c|c|c|c|c|}
\hline & \multicolumn{3}{|c|}{ Men $(\mathrm{N}=839)$} & \multicolumn{3}{|c|}{ Women (N=617) } \\
\hline & $\mathrm{N}$ & $\begin{array}{l}\text { Incidence } \\
(\%)\end{array}$ & $\begin{array}{c}P- \\
\text { value a }\end{array}$ & $N$ & $\begin{array}{l}\text { Incidence } \\
(\%)\end{array}$ & $\begin{array}{c}P- \\
\text { value a }\end{array}$ \\
\hline \multicolumn{2}{|l|}{$\begin{array}{l}\text { Lack of prior information } \\
\text { on amount of work to be } \\
\text { done each day }\end{array}$} & & 0.401 & & & $0.712^{b}$ \\
\hline No & 719 & 6.3 & & 589 & 7.5 & \\
\hline Yes & 117 & 4.3 & & 27 & 3.7 & \\
\hline $\begin{array}{l}\text { Variable weekly } \\
\text { working time }\end{array}$ & & & 0.465 & & & 0.284 \\
\hline No & 352 & 5.4 & & 314 & 8.3 & \\
\hline Yes & 483 & 6.6 & & 298 & 6.0 & \\
\hline Temporary employment & & & $0.356^{b}$ & & & $0.759 \mathrm{~b}$ \\
\hline No & 785 & 6.4 & & 574 & 7.5 & \\
\hline Yes & 50 & 2.0 & & 40 & 5.0 & \\
\hline \multicolumn{7}{|c|}{$\begin{array}{l}\text { Working postures and } \\
\text { biomechanical constraints }\end{array}$} \\
\hline $\begin{array}{l}\text { High repetitiveness of } \\
\text { tasks ( } \geq 4 \mathrm{~h} / \text { day) }\end{array}$ & & & 0.874 & & & 0.425 \\
\hline No & 664 & 6.2 & & 445 & 6.7 & \\
\hline Yes & 171 & 5.9 & & 162 & 8.6 & \\
\hline $\begin{array}{l}\text { High perceived physi- } \\
\text { cal exertion d }\end{array}$ & & & 0.030 & & & 0.315 \\
\hline No & 670 & 5.2 & & 487 & 6.8 & \\
\hline Yes & 164 & 9.8 & & 128 & 9.4 & \\
\hline \multicolumn{2}{|l|}{$\begin{array}{l}\text { Repeated and sustained } \\
\text { posture with the arms } \\
\text { above shoulder level } \\
\text { ( } \geq 2 \text { h/day) }\end{array}$} & & 0.004 & & & $0.202^{b}$ \\
\hline No & 743 & 5.3 & & 551 & 6.9 & \\
\hline Yes & 94 & 12.8 & & 62 & 11.3 & \\
\hline $\begin{array}{l}\text { Repeated and sus- } \\
\text { tained arm abduction } \\
\left(60-90^{\circ}\right)\end{array}$ & & & 0.844 & & & 0.003 \\
\hline No & 548 & 6.2 & & 457 & 5.5 & \\
\hline Yes & 290 & 5.9 & & 158 & 12.7 & \\
\hline \multicolumn{2}{|l|}{$\begin{array}{l}\text { Holding hand behind } \\
\text { the trunk ( } \geq 2 \mathrm{~h} / \text { day })\end{array}$} & & $0.429 b$ & & & $0.475^{b}$ \\
\hline No & 806 & 6.0 & & 586 & 7.2 & \\
\hline Yes & 31 & 9.7 & & 30 & 10.0 & \\
\hline $\begin{array}{l}\text { Use of handtools } \\
(\geq 2 \mathrm{~h} / \text { day })\end{array}$ & & & 0.928 & & & 0.250 \\
\hline No & 399 & 6.0 & & 388 & 6.4 & \\
\hline Yes & 438 & 6.2 & & 223 & 9.0 & \\
\hline \multicolumn{2}{|l|}{$\begin{array}{l}\text { Use of vibrating hand- } \\
\text { tools ( } \geq 2 \mathrm{~h} / \text { day) }\end{array}$} & & 0.609 & & & $0.652^{b}$ \\
\hline No & 680 & 5.9 & & 592 & 7.3 & \\
\hline Yes & 158 & 7.0 & & 20 & 10.0 & \\
\hline $\begin{array}{l}\text { Exposure to cold tem- } \\
\text { perature }(\geq 4 \mathrm{~h} / \text { day })\end{array}$ & & & $0.764^{b}$ & & & $0.061^{b}$ \\
\hline No & 785 & 6.2 & & 591 & 6.9 & \\
\hline Yes & 53 & 3.8 & & 21 & 19.1 & \\
\hline \multicolumn{7}{|l|}{$\begin{array}{l}\text { Psychosocial factors } \\
\text { at work }\end{array}$} \\
\hline $\begin{array}{l}\text { High psychological } \\
\text { demand }\end{array}$ & & & 0.091 & & & 0.524 \\
\hline No & 439 & 4.8 & & 305 & 7.9 & \\
\hline Yes & 395 & 7.6 & & 306 & 6.5 & \\
\hline Low skill discretion & & & 0.690 & & & 0.537 \\
\hline No & 436 & 6.2 & & 245 & 8.2 & \\
\hline Yes & 397 & 5.5 & & 366 & 6.8 & \\
\hline Low decision authority & & & 0.444 & & & 0.416 \\
\hline No & 591 & 5.6 & & 376 & 6.7 & \\
\hline Yes & 244 & 7.0 & & 238 & 8.4 & \\
\hline
\end{tabular}

Table 3. Continued

\begin{tabular}{|c|c|c|c|c|c|c|}
\hline & \multicolumn{3}{|c|}{ Men $(\mathrm{N}=839)$} & \multicolumn{3}{|c|}{ Women $(\mathrm{N}=617)$} \\
\hline & N & $\begin{array}{c}\text { Incidence } \\
(\%)\end{array}$ & $\begin{array}{c}\mathrm{P}- \\
\text { value a }\end{array}$ & N & $\begin{array}{l}\text { Incidence } \\
(\%)\end{array}$ & $\begin{array}{l}\mathrm{P}- \\
\text { value a }^{\text {a }}\end{array}$ \\
\hline $\begin{array}{l}\text { Low supervisor } \\
\text { support }\end{array}$ & & & 0.208 & & & 0.120 \\
\hline No & 511 & 5.3 & & 388 & 6.2 & \\
\hline Yes & 323 & 7.4 & & 218 & 9.6 & \\
\hline Low coworker support & & & 0.020 & & & 0.301 \\
\hline No & 687 & 5.2 & & 500 & 6.8 & \\
\hline Yes & 145 & 10.3 & & 103 & 9.7 & \\
\hline $\begin{array}{l}\chi^{2} \text { test. } \\
\text { Fisher exact test. } \\
\text { Lateral epicondylitis, } \\
\text { Quervain's disease an } \\
\text { the forearm-wrist regi }\end{array}$ & $x+$ & $\begin{array}{l}\text { el syndr } \\
\text { xtensor }\end{array}$ & $\begin{array}{l}\text { me, carp } \\
\text { eritendin }\end{array}$ & $\begin{array}{l}\text { al tun } \\
\text { itis or }\end{array}$ & $\begin{array}{l}\text { nnel syndror } \\
r \text { tenosynov }\end{array}$ & $\begin{array}{l}\text { me, De } \\
\text { vitis of }\end{array}$ \\
\hline
\end{tabular}

follow-up. For women, exposure to low coworker support, paced work, work pace dependent on automatic rate, work pace dependent on colleagues' work, and work with temporary workers were more frequent for the workers lost to follow-up. We observed no difference in terms of shoulder pain at baseline.

\section{Study population}

Analyses were based on 1456 workers ( 839 men and 617 women) without RCS at baseline (figure 1). More women $(31.2 \%)$ experienced shoulder pain at baseline than men $(25.9 \%), 8.6 \%$ of them declared symptoms lasting for $>1$ month during the preceding 12 months in the first questionnaire (versus $4.6 \%$ among men). The men worked mainly in the industry and services sectors, and the women in the services sector. The men were mainly skilled and unskilled blue-collar workers whereas the women were mainly low-grade white-collar workers (table 2).

\section{Diagnosis at follow-up}

RCS was diagnosed in 51 men (6.1\%) and 45 women (7.3\%), without statistical difference between genders. The right shoulder was involved in 52 cases, the left shoulder in 26 cases, and 18 cases were bilateral.

\section{Risk factors}

Bivariate analyses showed that personal characteristics, work organization, biomechanical factors, and psychosocial factors at work were associated with incident RCS (table 3).

For men, age was the strongest predictor of incident RCS, with an increased risk until 49 years of age. Work- 
Table 4. Multivariate model of risk factors for incident rotator cuff syndrome (RCS) in the male working population. ${ }^{\mathrm{a}}$

\begin{tabular}{|c|c|c|c|c|c|c|c|c|c|c|}
\hline & \multicolumn{10}{|c|}{ Men ( $\mathrm{N}=825 ; 51 \mathrm{RCS})$} \\
\hline & \multicolumn{5}{|c|}{ Model 1} & \multicolumn{5}{|c|}{ Model 2} \\
\hline & $\mathrm{N}$ & $\%$ & $\mathrm{OR}$ & $95 \% \mathrm{Cl}$ & P-value & $\mathrm{N}$ & $\%$ & $\mathrm{OR}$ & $95 \% \mathrm{Cl}$ & P-value \\
\hline Age & & & & & 0.001 & & & & & 0.001 \\
\hline$<40$ & 444 & 3.2 & 1 & & & 444 & 3.2 & 1 & & \\
\hline $40-44$ & 161 & 6.8 & 2.3 & $1.0-5.2$ & & 161 & 6.8 & 2.3 & $1.0-5.2$ & \\
\hline $45-49$ & 132 & 12.9 & 4.6 & $2.2-9.8$ & & 132 & 12.9 & 4.7 & $2.2-10.0$ & \\
\hline$\geq 50$ & 88 & 10.2 & 3.6 & $1.5-8.8$ & & 88 & 10.2 & 3.7 & $1.5-9.0$ & \\
\hline High perceived physical exertion ${ }^{b}$ & & & & & 0.163 & & & & & \\
\hline No & 662 & 5.3 & 1 & & & & & & & \\
\hline Yes & 163 & 9.8 & 1.6 & $0.8-3.2$ & & & & & & \\
\hline $\begin{array}{l}\text { Repeated and sustained posture with the arms } \\
\text { above shoulder level ( } \geq 2 \mathrm{~h} / \text { day })\end{array}$ & & & & & 0.043 & & & & & \\
\hline No & 732 & 5.3 & 1 & & & & & & & \\
\hline Yes & 93 & 12.9 & 2.2 & $1.0-4.7$ & & & & & & \\
\hline $\begin{array}{l}\text { High perceived physical exertionb and repeated and } \\
\text { sustained posture with the arms above shoulder } \\
\text { level ( } \geq 2 \mathrm{~h} / \text { day) }\end{array}$ & & & & & & & & & & 0.014 \\
\hline No factor & & & & & & 613 & 4.9 & 1 & & \\
\hline One factor & & & & & & 168 & 8.3 & 2.0 & $1.0-3.8$ & \\
\hline Both factors & & & & & & 44 & 15.9 & 3.3 & $1.3-8.4$ & \\
\hline Low coworker support & & & & & 0.033 & & & & & 0.035 \\
\hline No & 681 & 5.3 & 1 & & & 681 & 5.3 & 1 & & \\
\hline Yes & 144 & 10.4 & 2.0 & $1.1-3.9$ & & 144 & 10.4 & 2.0 & $1.1-3.9$ & \\
\hline
\end{tabular}

a Hosmer-Lemeshow goodness-of-fit test: $P=0.540$ in Model 1 and $P=0.901$ in Model 2.

${ }^{b}$ RPE Borg scale $\geq 15$.

ing with repeated and sustained posture with the arms above shoulder level ( $\geq 2$ hours/day) was associated with RCS. However, high perceived physical exertion was a confounding factor and kept in the final model (table 4, model 1). A variable combining arms above shoulder level and high perceived physical exertion was constructed (model 2). An elevated OR was observed for exposure to both factors. A total of seven men had incident RCS and worked with a combination of arms above shoulder level and high perceived physical exertion. Six of them were blue-collar workers (road haulage drivers,

Table 5. Multivariate model of risk factors for incident rotator cuff syndrome (RCS) in the female working population. ${ }^{a}$

\begin{tabular}{lrrrrrr}
\hline & \multicolumn{5}{c}{ Women (N=614; 45 RCS) } \\
\cline { 2 - 7 } & $\mathrm{N}$ & $\%$ & OR & $95 \% \mathrm{Cl}$ & P-value \\
\cline { 2 - 7 } Age & & & & & 0.002 \\
$<40$ & 297 & 3.7 & 1 & & \\
$40-44$ & 119 & 8.4 & 3.0 & $1.2-7.4$ & \\
$45-49$ & 116 & 10.3 & 3.4 & $1.4-8.0$ & \\
$\geq 50$ & 82 & 14.6 & 5.4 & $2.3-13.2$ & \\
$\quad$ Work with temporary workers & & & & & 0.016 \\
$\quad$ No & 448 & 5.8 & 1 & & \\
$\quad$ Yes & 166 & 11.5 & 2.2 & $1.2-4.2$ & \\
Repeated and sustained arm & & & & & 0.003 \\
abduction $\left(60-90^{\circ}\right)$ & & & & & \\
$\quad$ No & 457 & 5.5 & 1 & & \\
$\quad$ Yes & 157 & 12.7 & 2.6 & $1.4-5.0$ & \\
\hline
\end{tabular}

a Hosmer-Lemeshow goodness-of-fit test: $P=0.820$. skilled building maintenance workers, storekeepers, and mechanical machinery assemblers). In addition, low coworker support increased the risk of RCS.

For women, age was the strongest predictor of incident RCS. Both working with colleagues in temporary employment and repeated and sustained arm abduction $\left(60-90^{\circ}\right)$ were associated with RCS (table 5). A total of 19 women had incident RCS and worked with colleagues in temporary employment, and 8 of these were lower-grade white-collar workers (hospital cleaners, secretaries, shop cashiers, waitresses and bartenders, etc) and 8 were bluecollar workers (cleaners, chemical, rubber and plastic product assemblers, meat-and food-processing machine operators, shoe and leather workers, cleaners, etc). Most of them were in permanent employment (89.5\%). None of the factors related to the work organization remained in the final models for either gender.

\section{Sub-cohort without RCS and shoulder pain for more than one month at baseline}

In the sub cohort of workers without RCS and shoulder pain for $>1$ month at baseline (797 men and 551 women), RCS was diagnosed in 44 men (5.5\%) and 36 women $(6.5 \%)$, without statistical difference between genders. As shown in table 6, the risk factors remained the same. For men, low coworker support was of borderline of significance $(\mathrm{P}=0.066)$ as was working with colleagues in temporary employment $(\mathrm{P}=0.060)$ for women. 
Table 6. Multivariate model of risk factors for incident rotator cuff syndrome (RCS) in the subcohort of workers without shoulder pain for more than one month at baseline. a

\begin{tabular}{|c|c|c|c|c|c|c|c|c|c|c|}
\hline & \multicolumn{5}{|c|}{ Men (N=784; 44 RCS) } & \multicolumn{5}{|c|}{ Women (N=548; 36 RCS) } \\
\hline & $\mathrm{N}$ & $\%$ & $\mathrm{OR}$ & $95 \% \mathrm{Cl}$ & P-value & $\mathrm{N}$ & $\%$ & $\mathrm{OR}$ & $95 \% \mathrm{Cl}$ & P-value \\
\hline Age & & & & & $<0.001$ & & & & & 0.001 \\
\hline$<40$ & 428 & 2.6 & 1 & & & 279 & 2.5 & 1 & & \\
\hline $40-44$ & 154 & 6.5 & 2.7 & $1.1-6.4$ & & 106 & 4.7 & 2.1 & $0.7-6.1$ & \\
\hline $45-49$ & 119 & 12.6 & 5.8 & $2.5-13.1$ & & 92 & 5.4 & 3.4 & $1.3-9.0$ & \\
\hline$\geq 50$ & 83 & 9.6 & 4.3 & $1.7-11.3$ & & 66 & 13.6 & 6.4 & $2.5-16.3$ & \\
\hline Work with temporary workers & & & & & & & & & & 0.060 \\
\hline No & & & & & & 404 & 3.5 & 1 & & \\
\hline Yes & & & & & & 139 & 8.6 & 2.0 & $1.0-4.3$ & \\
\hline $\begin{array}{l}\text { High perceived physical exertion }{ }^{\mathrm{b}} \text { and } \\
\text { repeated and sustained posture with the arms } \\
\text { above shoulder level ( } \geq 2 \mathrm{~h} / \text { day) }\end{array}$ & & & & & 0.009 & & & & & \\
\hline 0 & 587 & 4.3 & 1 & & & & & & & \\
\hline 1 & 158 & 8.2 & 2.3 & $1.1-4.7$ & & & & & & \\
\hline 2 & 38 & 15.4 & 3.7 & $1.4-10.0$ & & & & & & \\
\hline Repeated and sustained arm abduction $\left(60-90^{\circ}\right)$ & & & & & & & & & & 0.001 \\
\hline No & & & & & & 409 & 3.9 & 1 & & \\
\hline Yes & & & & & & 134 & 7.5 & 3.3 & $1.6-6.9$ & \\
\hline Low coworker support & & & & & 0.066 & & & & & \\
\hline No & 655 & 4.9 & 1 & & & & & & & \\
\hline Yes & 129 & 9.3 & 2.0 & $1.0-4.0$ & & & & & & \\
\hline
\end{tabular}

${ }^{a}$ Hosmer-Lemeshow goodness-of-fit test: $P=0.490$ among men and $P=0.983$ among women.

${ }^{\mathrm{b}} \mathrm{RPE}$ Borg scale $\geq 15$.

\section{Discussion}

In this sample of workers free of RCS at baseline, $6.1 \%$ of men and $7.3 \%$ of women developed RCS. Age was the strongest predictor. High perceived physical exertion, working with repeated and sustained posture with the arms above shoulder level and low coworker support were also associated with incident RCS for men and working with colleagues in temporary employment and repeated and sustained arm abduction $\left(60-90^{\circ}\right)$ were associated with incident RCS for women.

There was no difference in incidence of RCS between men and women in this study. In some studies, the prevalence of positive responses to clinical tests did not differ according to gender $(3,9,27)$ but other studies have reported higher prevalence rates for men (1, 2). However, in our study, work-related factors differed according to gender. This gender difference probably reflects differences in exposure to work constraints. Several studies have reported that even when the job title is the same, the job content is different between men and women (27-29).

Age was the strongest predictor of incident RCS for both genders. This is consistent with knowledge regarding the degenerative changes in the rotator cuff tendons occurring during ageing $(9,12,30)$. Moreover, age was correlated with length of service, and an effect of cumulative exposure to biomechanical factors cannot be excluded. However length of service at baseline was not associated with RCS for either gender $(\mathrm{P}>0.20)$.

Recent studies have reported associations between clinically diagnosed shoulder disorders and personal factors, such as diabetes mellitus and waist and hip circumference $(7,9,10,12)$. Due to the small number of workers with diabetes mellitus, we could not study this factor. We did not find any association between BMI and incident RCS.

Working with repeated and sustained posture with the arms above the shoulder was the main biomechanical risk factor for incident RCS among men. Strong biomechanical evidence supports this finding since the main pathophysiological mechanisms of RCS are compression of the tendons between the humeral head and the coracoacromial arch and ischemia due to impingement or increased intramuscular pressure following extreme arm abduction $(8,30)$. The same result was reported for the prevalent cases at baseline (7), in agreement with other cross-sectional studies $(3,10)$. Svenden et al (11) reported that working with the arms in a highly elevated position was associated with MRI-diagnosed alterations in the supraspinatus tendon. Moreover, in our study the strength of this association increased when working with arms above the shoulder was combined with high perceived physical exertion. Silverstein et al (3) showed that the combination of upper arm flexion $\geq 45^{\circ}$ and forceful exertion was a significant risk factor for RCS. Repeated and sustained arm abduction (60-90 $)$ 
was associated with incident RCS for women, as in the analysis of prevalent cases (7).

Working with temporary workers increased the risk of incident RCS in women. This mostly involved women in permanent employment, and we could hypothesize that the working load of such experienced women was increased because they may have had to spend part of their working time training less qualified colleagues in temporary work.

We found an association between incident RCS and low coworker support for men. Kaergaard et al (4) reported that low social support (from colleagues and supervisors) was associated with the development of a neck-shoulder disorder among female sewing machine operators. Other studies of RCS have not shown an association with psychosocial factors, and none of the dimensions of the Karasek Job Content Questionnaire have been shown to be more significant than any other (31).

A total of $56.6 \%$ of the baseline cohort were not able to be followed up for physical examination. Of these, $58.5 \%$ were no longer being monitored by any OP of the network, because they had left their baseline jobs without informing their OP or because their OP had refused to participate. The follow-up period coincided with a major economic crisis in the region during 2008-2009, when the regional salaried workforce declined by $3.4 \%$, and even by $33.7 \%$ in temporary employment agencies according to the French Economic Institute (32).

The lowest participation rate in this study was among young workers, workers in temporary employment at baseline, and those with a short length of service at baseline. This was to be expected because of the difficulty of following up young workers in insecure employment. According to the literature, these workers often present fewer upper-extremity MSD, and this can lead to overestimation of the incidence of upper-extremity MSD. This was amplified by the economic crisis, which strongly affected temporary employment and younger workers. Workers with a risk factor for upper-extremity MSD at baseline generally less often underwent the second physical examination, even though workers in the industrial sector in France have more frequent mandatory physical examinations. We suspect that the economic crisis may have excluded the workers most exposed to the risk of upper-extremity MSD from work (and from follow-up), including workers in industry. A study on the impact of loss to follow-up in epidemiological studies on upper-extremity MSD found (33) that the differences in the characteristics between participants and those lost to follow-up did not influence the risk ratios for associations between exposure factors for upper-extremity MSD and upper-extremity MSD status. We therefore believe that there was no major selection phenomenon associated with the quality of the follow-up.
One of the limitations of our study was that we had no medical information between baseline and follow-up, so the cases occurring between the two examinations who were negative at follow-up were not considered as "incident cases" in our analyses.

The design of the study meant that only the subjects still at work were included in the cohort. Indeed OP performed the physical examination, and they saw only employed people. A "healthy worker effect" may thus have occurred, meaning that subjects with RCS were more representative of milder RCS than severe RCS.

Trained OP clinically assessed RCS using a physical examination including standardized provocation tests (23). Due to cost and time limitations, direct exposure measurements by observation were not possible in this surveillance program. A self-administered questionnaire was used to assess the work-related factors. Answers were requested for a typical workday in the preceding 12-month period to limit recall errors (34), and awkward postures were presented in picture form to facilitate workers' understanding and increase the validity of self-assessment of postures. As far as possible, standardized and validated instruments (such as the European consensus for biomechanical factors and the Karasek Job Content Questionnaire for psychosocial factors) were used $(23,26)$. However, the within- and between variability of posture were not assessed, and we cannot exclude the possibility that self-reporting of exposure may have biased risk estimates, since workers experiencing shoulder pain at baseline may overrate their exposure levels (35). However, in the analysis without workers with chronic shoulder pain at baseline, the same risk factors were identified.

Some studies have reported physical exercise as a risk factor for incident shoulder pain $(12,36-38)$. Due to the length of our self-administered questionnaire, we preferred not to ask questions about sports, hobbies or life events. Although non-work activities may represent important confounders, we believe that their influence is probably not sufficient to affect the value of the study.

In conclusion, this study showed the multifactorial origin of incident RCS and highlighted a limited number of personal and work-related risk factors, differing according to gender. The effect of age was greater than that of work-related factors. Arm abduction was the major work-related risk factor. A psychosocial factor was significant only for men. Since age is not modifiable, mechanical and psychosocial exposure should be an important target for strategies for the prevention of RCS in the working population. 


\section{Acknowledgements}

This project was supported by grants from the French Institute for Public Health Surveillance, Saint-Maurice, France (grant 9/25/2002-5 "Réseau expérimental de surveillance des troubles musculo-squelettiques") and the French National Research Agency (ANR-grant SEST06-36). We thank the occupational physicians involved in the sentinel network: Doctors Abonnat, Adam, Addou, Agullo, Ansaloni, Aubrun, Banon, Bardet, Barraya, Beaurepaire, Becquemie, Berthelot, Bertin, Bertrand, Bidron, Biton, Biziou-Fouere, Bizouarne, Boisse, Bonamy, Bonneau, Bouchet, Bouguer, Bourrut-Lacouture, Bourven, Bradane, Breton, Bricaud, Caillon, Camer, Cesbron, Chabot, Charlon, Chevalier, Chisacof, Chotard, Clement dit Pontieu, Compain, Coquin-Georgeac, Cordes, Cormier, Couet, Coutand, Da Costa, Dachert, Dadourian, Danielou, Darcy, Davenas, De Lansalut, De Lescure, Diquelou, Dopsent, Dubois, Dufrenne-Benetti, Dupas, Durand, Durand-Perdriel, Evano, Fache, Faline, Fontaine, Fosse, Frampas-Chotard, François, Garrabe, Gasseau, Giffard, Girard, Girardin, Guerin, Guessard, Guillaumin, Guillier, Guillimin, Guinel, Harinte, Harrigan, Hefti, Herrouet, Herson, Hervio, Hirigoyen, Houssin, Husquin, Jahan, Jarry, Jube, Kalfon, Kergresse, Khouri, Krai, Labraga, Laine, Laine-Colin, Lamotte, Lasnier, Laventure, Le Clerc, Le Dizet, Le Mauff, Lechevalier, Lecompte, Ledenvic, Leroux, Leroy-Maguer, Levrard, Levy, Ligeard, Logeay, Louineau, Lourtis, Lucas, Maeker, Maison, Mallet, Marquiset, Martin, Martin-Laurent, Mazoyer, Meritet, Meyer, Michel MC, Michel R, MigneCousseau, Moisan, Morvan, Mouchet, Moui, Nivet, Page, Parrot, Patillot, Perou, Pierfitte, Pinaud, Pineau, Pizzalla, Plessis, Plouhinec, Pocreaux, Prod'homme, Puichaud, Quince, Rabjeau, Raffray, Riberot, Riou, Robin, RobinRiom, Roesch, Rouault, Roussel, Roux, Russu, Saboureault, Schlindwein, Soulard, Souvre-Debray, Spiesser, Thomas, Thomasset, Thomson, Tillette, Treillard, Tripodi, Verrier, Voisin.

\section{References}

1. Miranda H, Punnett L, Viikari-Juntura E, Heliövaara M, Knekt P. Physical work and chronic shoulder disorder. Results of a prospective population-based study. Ann Rheum Dis. 2008;67(2):218-23. http://dx.doi.org/10.1136/ ard.2007.069419.

2. Silverstein BA, Viikari-Juntura E, Fan ZJ, Bonauto DK, Bao S, Smith C. Natural course of nontraumatic rotator cuff tendinitis and shoulder symptoms in a working population. Scand J Work Environ Health. 2006;32(2):99-108. http://dx.doi. org/10.5271/sjweh. 985 .
3 Silverstein BA, Bao SS, Fan ZJ, Howard N, Smith C, Spielholz $\mathrm{P}$, et al. Rotator cuff syndrome: personal, work-related psychosocial and physical load factors. J Occup Environ Med. 2008;50(9):1062-76. http://dx.doi.org/10.1097/ JOM.0b013e31817e7bdd.

4. Kaergaard A, Andersen JH. Musculoskeletal disorders of the neck and shoulders in female sewing machine operators: prevalence, incidence, and prognosis. Occup Environ Med. 2000;57(8):528-34. http://dx.doi.org/10.1136/oem.57.8.528.

5. Bonde JP, Mikkelsen S, Andersen JH, Fallentin N, Baelum J, Svendsen SW, et al. Prognosis of shoulder tendonitis in repetitive work: a follow up study in a cohort of Danish industrial and service workers. Occup Environ Med. 2003;60(9):E8. http://dx.doi.org/10.1136/oem.60.9.e8.

6. Walker-Bone K, Palmer KT, Reading I, Coggon D, Cooper C. Prevalence and impact of musculoskeletal disorders of the upper limb in the general population. Arthritis Rheum. 2004;51(4):642-51. http://dx.doi.org/10.1002/art.20535.

7. Roquelaure Y, Bodin J, Ha C, Petit Le Manac'h A, Descatha A, Chastang J-F, et al. Personal, biomechanical, and psychosocial risk factors for rotator cuff syndrome in a working population. Scand J Work Environ Health. 2011;37(6):502-11. http:// dx.doi.org/10.5271/sjweh.3179.

8. Hagberg M, Silverstein B, Wells R, Smith M, Hendrick H, Carayon P, et al. Work related musculoskeletal disorders (WMSDs): a reference book for prevention. London: Taylor \& Francis; 1995

9. Rechardt M, Shiri R, Karppinen J, Jula A, Heliövaara M, Viikari-Juntura E. Lifestyle and metabolic factors in relation to shoulder pain and rotator cuff tendinitis: a population-based study. BMC Musculoskelet Disord. 2010;11:165. http:// dx.doi.org/10.1186/1471-2474-11-165.

10. Miranda H, Viikari-Juntura E, Heistaro S, Heliövaara M, Riihimäki H. A population study on differences in the determinants of a specific shoulder disorder versus nonspecific shoulder pain without clinical findings. Am J Epidemiol. 2005;161(9):847-55. http://dx.doi.org/10.1093/aje/kwi112.

11. Svendsen SW, Gelineck J, Mathiassen SE, Bonde JP, Frich LH, Stengaard-Pedersen K, et al. Work above shoulder level and degenerative alterations of the rotator cuff tendons: a magnetic resonance imaging study. Arthritis Rheum. 2004;50(10):3314 22. http://dx.doi.org/10.1002/art.20495.

12. Viikari-Juntura E, Shiri R, Solovieva S, Karppinen J, LeinoArjas P, Varonen H, et al. Risk factors of atherosclerosis and shoulder pain - Is there an association? A systematic review. Eur J Pain. 2008;12(4):412-26. http://dx.doi.org/10.1016/j. ejpain.2007.08.006.

13. Wendelboe AM, Hegmann KT, Gren LH, Alder SC, White GL Jr, Lyon JL. Associations between body-mass index and surgery for rotator cuff tendinitis. J Bone Joint Surg Am. 2004;86-A(4):743-7.

14. Frost P, Andersen JH. Shoulder impingement syndrome in relation to shoulder intensive work. Occup Environ Med. 1999;56(7):494-8. http://dx.doi.org/10.1136/oem.56.7.494

15. Sutinen P, Toppila E, Starck J, Brammer A, Zou J, Pyykkö I. 
Hand-arm vibration syndrome with use of anti-vibration chain saws: 19-year follow-up study of forestry workers. Int Arch Occup Environ Health. 2006;79(8):665-71. http://dx.doi. org/10.1007/s00420-006-0099-2.

16. Svendsen SW, Bonde JP, Mathiassen SE, Stengaard-Pedersen $\mathrm{K}$, Frich LH. Work related shoulder disorders: quantitative exposure-response relations with reference to arm posture. Occup Environ Med. 2004;61(10):844-53. http://dx.doi. org/10.1136/oem.2003.010637.

17. Malchaire J, Cock N, Vergracht S. Review of the factors associated with musculoskeletal problems in epidemiological studies. Int Arch Occup Environ Health. 2001;74(2):79-90. http://dx.doi.org/10.1007/s004200000212.

18. Bernard B. Musculoskeletal disorders and workplace factors. A critical review of epidemiologic evidence for work-related musculoskeletal disorders of the neck, upper-extremity, and low back. NIOSH. Cincinnati. 1997;DHHS (NIOSH) Publication.

19. van Rijn RM, Huisstede BM, Koes BW, Burdorf A. Associations between work-related factors and specific disorders of the shoulder--a systematic review of the literature. Scand J Work Environ Health. 2010;36(3):189-201. http:// dx.doi.org/10.5271/sjweh.2895.

20. Ha C, Roquelaure Y, Leclerc A, Touranchet A, Goldberg M, Imbernon E. The French Musculoskeletal Disorders Surveillance Program: Pays de la Loire network. Occup Environ Med. 2009;66(7):471-9. http://dx.doi.org/10.1136/ oem.2008.042812.

21. Roquelaure Y, Ha C, Leclerc A, Touranchet A, Sauteron M, Melchior M, et al. Epidemiologic surveillance of upper-extremity musculoskeletal disorders in the working population. Arthritis Rheum. 2006;55(5):765-78. http:// dx.doi.org/10.1002/art.22

22. Roquelaure $\mathrm{Y}, \mathrm{Ha} \mathrm{C}$, Rouillon $\mathrm{C}$, Fouquet $\mathrm{N}$, Leclerc A, Descatha A, et al. Risk factors for upperextremity musculoskeletal disorders in the working population. Arthritis Rheum. 2009;61(10):1425-34. http://dx.doi.org/10.1002/art.24740.

23. Sluiter JK, Rest KM, Frings-Dresen MH. Criteria document for evaluating the work-relatedness of upper-extremity musculoskeletal disorders. Scand J Work Environ Health. 2001;Suppl 1:1-102.

24. Borg G. Psychophysical scaling with applications in physical work and the perception of exertion. Scand J Work Environ Health. 1990;Suppl 1:55-8.

25. Jang R, Karwowski W, Quesada PM, Rodrick D, Sherehiy B, Cronin SN, et al. Biomechanical evaluation of nursing tasks in a hospital setting. Ergonomics. 2007;50(11):1835-55. http:// dx.doi.org/10.1080/00140130701674661.

26. Niedhammer I, Chastang JF, Gendrey L, David S, Degioanni S. [Psychometric properties of the French version of Karasek's "Job Content Questionnaire" and its scales measuring psychological pressures, decisional latitude and social support: the results of the SUMER]. Sante Publique. 2006;18(3):41327. http://dx.doi.org/10.3917/spub.063.0413.
27. Silverstein B, Fan ZJ, Smith CK, Bao S, Howard N, Spielholz $\mathrm{P}$, et al. Gender adjustment or stratification in discerning upper extremity musculoskeletal disorder risk? Scand J Work Environ Health. 2009;35(2):113-26. http://dx.doi. org/10.5271/sjweh.1309.

28. Feveile H, Jensen C, Burr H. Risk factors for neck-shoulder and wrist-hand symptoms in a 5-year follow-up study of 3,990 employees in Denmark. Int Arch Occup Environ Health. 2002;75(4):243-51. http://dx.doi.org/10.1007/s00420-0010298-9.

29. de Zwart BC, Frings-Dresen MH, Kilbom A. Gender differences in upper extremity musculoskeletal complaints in the working population. Int Arch Occup Environ Health. 2001;74(1):21-30. http://dx.doi.org/10.1007/ s004200000188.

30. Järvholm U, Styf J, Suurkula M, Herberts P. Intramuscular pressure and muscle blood flow in supraspinatus. Eur J Appl Physiol Occup Physiol. 1988;58(3):219-24. http://dx.doi. org/10.1007/BF00417252.

31. Bongers PM, Ijmker S, van den Heuvel S, Blatter BM. Epidemiology of work related neck and upper limb problems: psychosocial and personal risk factors (part I) and effective interventions from a bio behavioural perspective (part II). J Occup Rehabil. 2006;16(3):279-302. http://dx.doi. org/10.1007/s10926-006-9044-1.

32. INSEE. Crise en Pays de la Loire : industrie et intérim dans la tourmente. INSEE. 2010;(88):1.

33. Bildt C, Alfredsson L, Punnett L, Theobald H, Torgén M, Wikman A. Effects of drop out in a longitudinal study of musculoskeletal disorders. Occup Environ Med. 2001;58(3):194-9. http://dx.doi.org/10.1136/oem.58.3.194.

34. Miranda H, Gold JE, Gore R, Punnett L. Recall of prior musculoskeletal pain. Scand J Work Environ Health. 2006;32(4):294-9. http://dx.doi.org/10.5271/sjweh.1013.

35. Punnett L, Wegman DH. Work-related musculoskeletal disorders: the epidemiologic evidence and the debate. J Electromyogr Kinesiol. 2004;14(1):13-23. http://dx.doi. org/10.1016/j.jelekin.2003.09.015.

36. Miranda H, Viikari-Juntura E, Martikainen R, Takala EP, Riihimäki H. A prospective study of work related factors and physical exercise as predictors of shoulder pain. Occup Environ Med. 2001;58(8):528-34. http://dx.doi.org/10.1136/ oem.58.8.528.

37. Cassou B, Derriennic F, Monfort C, Norton J, Touranchet A. Chronic neck and shoulder pain, age, and working conditions: longitudinal results from a large random sample in France. Occup Environ Med. 2002;59(8):537-44. http://dx.doi. org/10.1136/oem.59.8.537.

38. Siivola SM, Levoska S, Latvala K, Hoskio E, Vanharanta $\mathrm{H}$, Keinänen-Kiukaanniemi S. Predictive factors for neck and shoulder pain: a longitudinal study in young adults. Spine. 2004;29(15):1662-9. http://dx.doi.org/10.1097/01. BRS.0000133644.29390.43.

Received for publication: 14 December 2012 